\title{
Amélioration de la disponibilité du phosphore par la gestion de la macrofaune du sol : cas d'un lixisol en zone semi-aride du Burkina Faso
}

\author{
Jean OUEDRAOGO ${ }^{1 *}$, Hassan Bismarck NACRO ${ }^{1}$, Elisée OUEDRAOGO ${ }^{2}$, \\ Sansan YOUL $^{3}$ et Michel P. SEDOGO ${ }^{4}$ \\ ${ }^{1}$ Institut du Développement Rural, Laboratoire d'Etude et de Recherche sur la Fertilité des Sols (LERF), \\ Université Polytechnique de Bobo-Dioulasso, 01 BP 1091 Bobo-Dioulasso, Burkina Faso. \\ 2 Banque Mondiale, 01 BP 622 Ouagadougou 01, Burkina Faso. \\ ${ }^{3}$ International Fertilizer Development Center, 11 BP 82 Ouagadougou 11, Burkina Faso. \\ ${ }^{4}$ Institut de l'Environnement et de Recherches Agricoles (INERA), \\ 04 BP 8645 Ouagadougou 04, Burkina Faso. \\ *Auteur correspondant ; E-mail: jeanouedraogo84@yahoo.fr; Tel. (+226) 70634360.
}

\section{RESUME}

La carence en phosphore des sols de l'Afrique de l'Ouest constitue une contrainte majeure à l'intensification des systèmes de production agricole. Cette étude a été conduite pour évaluer les effets de l'interaction entre la macrofaune et des technologies de gestion de la fertilité des sols sur la disponibilité du phosphore d'un lixisol en zone semi-aride du Burkina Faso. Un dispositif expérimental en split-plot comprenant deux traitements principaux (parcelles avec et sans macrofaune) et cinq technologies de gestion de la fertilité des sols a été utilisé. La disponibilité du phosphore a été évaluée en utilisant la méthode Bray I. Les résultats montrent qu'il y a une interaction positive entre les technologies de gestion de la fertilité et la macrofaune du sol. La présence de la macrofaune a entraîné une augmentation significative du phosphore assimilable du sol. De même, l'apport de compost seul ou combiné à l'urée, augmente significativement la teneur en phosphore assimilable du sol. Ces résultats suggèrent de repenser les stratégies de protection des cultures, en réduisant et en utilisant de façon judicieuse les pesticides chimiques de synthèse. Les producteurs doivent en effet, adopter les biopesticides et les pesticides naturels afin de préserver la diversité biologique des sols agricoles.

(C) 2014 International Formulae Group. All rights reserved.

Mots clés : Macrofaune, qualité de la matière organique, phosphore assimilable, interaction Burkina Faso.

\section{INTRODUCTION}

Le phosphore, à l'instar de l'azote et du potassium, est un élément essentiel pour le règne végétal. Il joue un rôle primordial dans la croissance et le développement, ainsi que dans le métabolisme des plantes et le transport de l'énergie. Or, les sols de l'Afrique de l'Ouest en général sont carencés en phosphore
(Lompo et al., 2009). Cette déficience en phosphore des sols est une contrainte majeure à l'intensification de la productivité des sols (Lompo et al., 2009; Soma, 2010). Pour corriger cette déficience en éléments nutritifs en général et en phosphore en particulier, plusieurs modes de gestion de la fertilité combinant l'usage de la matière organique et 
des engrais minéraux ou de phosphate naturel ont alors été adoptés (Ouédraogo et al., 2005 ; Lompo, 2009). Toutefois, les rendements des cultures restent faibles. Ainsi, face à la baisse continue de la fertilité des sols, de la productivité des cultures, à la raréfaction des terres et à l'augmentation du prix des facteurs de production, l'Afrique de l'Ouest doit concevoir une agriculture plus productive et plus durable (Petit, 2011 ; Vall et al., 2012). Par conséquent, l'intensification écologique constitue une alternative pour améliorer durablement les rendements. Elle met l'accent sur l'utilisation de la régulation biologique dans la gestion des agroécosystèmes (Doré et al., 2011). Dans ce contexte, la macrofaune du sol, qui joue un rôle déterminant dans les divers processus physico-chimiques et biologiques des sols (Ouédraogo et al., 2005 ; Lavelle et al., 2006 ; Li et al., 2006) pourrait occuper une place centrale.

Des études ont déjà été faites sur les effets de la matière organique sur la macrofaune du sol (Zida et al., 2011 ; Traoré, 2012), les caractéristiques physico-chimiques $\mathrm{du}$ sol et les rendements des cultures (Ouédraogo et al., 2005 ; Lompo, 2009 ; Kiba et al., 2012). En réalisant une analyse chimique comparée des turricules de vers de terre et du sol, (Ouédraogo et al., 2005) ont montré que les vers de terre amélioraient la disponibilité du phosphore, du phosphate naturel (Burkina phosphate) en présence de compost ou de fumier riche. Chapuis-Lardy et al. $(2009$; 2011) ont également montré que les turricules de vers de terre augmentaient la disponibilité du phosphore. Les interactions entre les technologies de gestion de la fertilité et la macrofaune, pourraient être donc une clé pour améliorer la disponibilité du phosphore dans le sol. C'est dans ce cadre que s'inscrit cette étude dont l'objectif est d'évaluer l'effet de l'interaction de la macrofaune et des technologies de gestion des sols utilisées en milieu paysan sur la disponibilité du phosphore, afin de mieux définir des stratégies de fertilisation.

\section{MATERIEL ET METHODES Description du site}

Le dispositif expérimental a été installé au cours de la campagne agricole 2011-2012 sur un lixisol (WRB, 2006) en jachère depuis une quinzaine d'année, en zone Nord soudanienne du Burkina Faso (entre $12^{\circ} 08^{\prime}$ 02 " de latitude Nord et $1^{\circ} 24^{\prime} 54^{\prime \prime}$ de longitude Ouest). Les caractéristiques physico-chimiques de l'horizon $0-20 \mathrm{~cm}$ montrent qu'il s'agit d'un sol à texture limono-sableuse en surface $(12 \%$ d'argiles, $24 \%$ de limons et $65 \%$ de sables), acide ( $\mathrm{pH}-$ eau $=6,16$ ) et pauvre en matière organique $(0,97 \%)$. La teneur en phosphore assimilable du sol était de $1,3 \mathrm{mg} \mathrm{P.} \mathrm{kg}^{-1}$. La saison des pluies couvre les mois de juin à septembre avec une pluviosité moyenne de $749 \mathrm{~mm}$ pour les dix dernières années (2003-2012).

\section{Dispositif expérimental}

Un dispositif expérimental split-plot à trois répétitions a été utilisé. Il comprend deux (02) traitements principaux (parcelles avec macrofaune et parcelles sans macrofaune), cinq (05) traitements secondaires qui sont les différentes technologies de gestion de la fertilité du sol, résultant de la combinaison de deux substrats organiques de qualité contrastée avec l'urée et une parcelle témoin : compost (enfouissement de $4 \mathrm{t} \mathrm{MS} /$ ha de compost) ; compost + urée (enfouissement de $4 \mathrm{t}$ MS / ha de compost + de l'urée à la dose de $30 \mathrm{~kg} \mathrm{~N} /$ ha); tiges + urée (enfouissement de $4 \mathrm{t} \mathrm{MS} /$ ha de tiges de sorgho + de l'urée à la dose de $30 \mathrm{~kg} \mathrm{~N} / \mathrm{ha}$ ); tiges (enfouissement de $4 \mathrm{t}$ MS / ha de tiges de sorgho) et le témoin. La matière organique dont les caractéristiques sont présentées dans le Tableau 1 a été appliquée 3 jours avant le semis et toute l'urée a été appliquée au semis. Les parcelles élémentaires ont une superficie de $25 \mathrm{~m}^{2}(5 \mathrm{~m}$ x $5 \mathrm{~m}$ ). Une allée principale de $3 \mathrm{~m}$ sépare deux blocs consécutifs. Dans chaque bloc, les traitements principaux sont séparés par des allées secondaires de $2 \mathrm{~m}$, et les traitements secondaires par des allées de $1 \mathrm{~m}$. 
Le compost a été produit à l'aide de litière et de tiges de maïs par la technique du compostage aérobie en fosse, avec un retournement périodique de 15 jours pendant 3 mois.

\section{Destruction de la macrofaune du sol}

La destruction de la macrofaune a été réalisée par application au sol de pesticides chimiques de synthèse. Deux matières actives: le Chlorpyrifos-éthyl, (DADYRSBAN 4 E) à la dose de 240 g.ha $^{-1}$, et le friponil (HERCULES 50 SC) à la dose de 0,125 g.ha ${ }^{-1}$ ont été appliqués au semis, 16 jours après semis (JAS) et 42 JAS. Pour ce faire, un mélange contenant $2,5 \mathrm{ml}$ des pesticides HERCULES $50 \mathrm{SC}$ et DADYRSBAN $4 \mathrm{E}$ a été dilué dans 15 litres d'eau, puis appliqué sur chaque parcelle élémentaire à l'aide d'un pulvérisateur à dos et à pression soutenue.

\section{Echantillonnage de sol}

Le prélèvement des échantillons de sols a été fait à 48 JAS au moment de la floraison du niébé. Dans chaque parcelle élémentaire, un échantillon composite a été constitué à partir de cinq (05) échantillons élémentaires prélevés dans l'aire utile. Les échantillons de sols ont servi à la détermination du phosphore assimilable selon la méthode Bray I et du phosphore total.

\section{Analyses statistiques}

Les résultats du phosphore total et du phosphore assimilable du sol ont été soumis à une analyse de variance (ANOVA), avec le logiciel Genstat version Discovery 4. La séparation des moyennes a été effectuée par le test de la plus petite différence significative au seuil de $5 \%$.

L'interaction entre la macrofaune et les modes de gestion de la fertilité sur le phosphore assimilable a été calculée en utilisant la formule utilisée par (Ouédraogo et al., 2007). $\triangle$ Pass $F M(i)=\triangle$ PassF $+\triangle$ Pass $M(i)+\triangle$ Passint

Où:

$\triangle$ Pass FM (i) est l'effet de la macrofaune du sol et des technologies de gestion de la fertilité sur le phosphore assimilable du sol ; $\Delta$ Pass $\mathrm{F}$ est la contribution absolue de la macrofaune du sol ;

$\triangle$ Pass M (i) est la contribution absolue des technologies de gestion de la fertilité des sols ; $\Delta$ Passint est la contribution de l'interaction entre la macrofaune du sol et les technologies de gestion de la fertilité.

$\triangle$ Pass $F=$ PassF To - PassNF To

$\Delta$ PassM $(\mathrm{i})=$ PassNF $(\mathrm{i})-$ PassNF To

$\Delta$ Passint $=($ PassF $(\mathrm{i})-$ PassNF $(\mathrm{i}))-($ PassF To - PassNF To $)$

PassF (i) est le phosphore assimilable dans la parcelle i avec macrofaune du sol;

PassNF (i) est le phosphore assimilable dans la parcelle i sans macrofaune du sol;

PassNF To est le phosphore assimilable dans le témoin sans macrofaune du sol;

et PassF To, est le phosphore assimilable dans le témoin avec macrofaune du sol.

\section{RESULTATS}

\section{Macrofaune et disponibilité du phosphore dans le sol}

En présence de la macrofaune du sol, la teneur moyenne en phosphore assimilable est de 6,52 mg P. kg ${ }^{-1}$; elle baisse de 40,34\% en absence de la macrofaune du sol (Tableau 2). Les résultats de l'analyse de variance (Tableau 2) ont montré que la macrofaune du sol a influencé de façon significative la quantité de phosphore assimilable dans le sol. Par contre, elle n'a pas eu d'influence significative sur le phosphore total du sol.

\section{Modes de gestion de la fertilité des sols et disponibilité du phosphore}

La plus forte teneur en phosphore total (75,9 mg P. kg $\left.{ }^{-1}\right)$ a été obtenue avec la technologie à base de compost seul; elle est suivie du témoin $\left(70,2 \mathrm{mg} \mathrm{P} . \mathrm{kg}^{-1}\right)$. La plus faible teneur a été obtenue avec la technologie à base de tiges. Toutefois, l'analyse de 
variance ne révèle aucune différence significative entre la teneur en phosphore total des différentes technologies de gestion de la fertilité des sols (Tableau 2).

Les teneurs en phosphore assimilable (Bray I) ont varié significativement en fonction des technologies de gestion de la fertilité des sols (Tableau 2). La plus forte teneur en phosphore assimilable a été obtenue avec la technologie à base de compost seul $\left(6,22 \mathrm{mg} \mathrm{P} . \mathrm{kg}^{-1}\right)$. Elle est significativement différente de toutes les autres à l'exception des technologies combinant le compost et l'urée. L'apport de compost, seul ou en combinaison avec l'urée, entraîne donc une amélioration de la disponibilité du phosphore dans le sol.

Interaction macrofaune-modes de gestion de la fertilité, et disponibilité du phosphore assimilable

Dans les parcelles non traitées aux pesticides (présence de la macrofaune du sol), les plus fortes teneurs en phosphore assimilable sont observé sur les technologies à base de compost et de compost + urée avec respectivement 7,68 mg P. $\mathrm{kg}^{-1}$ et 7,3 mg P.kg 1. La plus faible teneur a été observée sur le témoin avec 5,37 mg P.kg- (Figure 1). L'apport de compost, et de compost + urée, a augmenté la teneur en phosphore assimilable respectivement de $30 \%$ et $26 \%$ par rapport au témoin. L'ajout d'urée aux tiges a entraîné une amélioration de la teneur en phosphore assimilable. Dans les parcelles traitées aux pesticides (Figure 1), une faible variation de la teneur en phosphore assimilable a été observée entre les différentes technologies de gestion de la fertilité des sols. La plus forte teneur (4,76 mg P. $\left.\mathrm{kg}^{-1}\right)$ a été observée avec la technologie à base de compost seul, et la plus faible a été observée avec les tiges (3,03 mg P. $\mathrm{kg}^{-1}$ ).

L'absence de la macrofaune entraîne une réduction la teneur en phosphore assimilable de $22,35 \%$ (parcelle témoin), à 49,08\% (parcelle avec apport de tige + urée). Sans apport de fertilisant, la contribution absolue de la macrofaune au phosphore assimilable du sol est de 1,2 mg P. $\mathrm{kg}^{-1}$. Cette contribution a été supérieure à celle des technologies de gestion de la fertilité des sols. En effet, l'apport de tiges seules, et la combinaison des tiges et de l'urée, ont eu un effet dépressif sur le phosphore assimilable du sol. Par contre, l'apport de compost a eu un effet positif (Figure 2).

L'analyse de variance n'a pas révélée d'effet significatif de l'interaction entre les technologies de gestion de la fertilité et la macrofaune sur le phosphore. Toutefois, une interaction positive entre la macrofaune et les technologies de gestion de la fertilité des sols sur le phosphore assimilable, a été observée (Figure 2). La plus forte interaction a été observée avec la technologie qui combine les

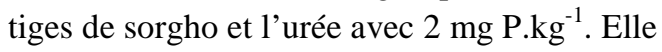
est suivie de la technologie qui combine compost et l'urée (1,92 mg P. $\left.\mathrm{kg}^{-1}\right)$. La plus faible interaction a été observée avec les tiges utilisées seules (1,53 mg P. $\left.\mathrm{kg}^{-1}\right)$.

Tableau 1 : Caractéristiques chimiques du compost et des tiges.

\begin{tabular}{lcc}
\hline Paramètres mesurés & Compost & Tiges \\
\hline Carbone total \% & 10,82 & 55,32 \\
Azote total \% & 0,533 & 0,2 \\
C/N & 20,3 & 276,6 \\
Phosphore total $(\mathrm{g} \mathrm{P} / \mathrm{kg})$ & 3,94 & 0,14 \\
Potassium total $(\mathrm{g} \mathrm{K} / \mathrm{Kg})$ & 3,23 & 6,66 \\
\hline
\end{tabular}


Tableau 2 : Effet de la macrofaune, des technologies de gestion de ka fertilité et de la macrofaune sur le phosphore total et assimilable du sol.

\begin{tabular}{lccc}
\hline Facteurs & Traitements & $\begin{array}{c}\text { Phosphore total } \\
\left(\mathbf{m g ~ P . k g}^{-1}\right)\end{array}$ & $\begin{array}{c}\text { Phosphore assimilable } \\
\left(\mathbf{m g ~ P . k k ^ { - 1 } )}\right.\end{array}$ \\
\hline & Parcelles avec macrofaune & $69,1^{\mathrm{a}}$ & $6,52^{\mathrm{a}}$ \\
Macrofaune & Parcelles sans macrofaune & $67,3^{\mathrm{a}}$ & $3,89^{\mathrm{b}}$ \\
& Probabilité & 0,675 & 0,006 \\
& Signification & $\mathrm{NS}$ & $\mathrm{HS}$ \\
& Coefficient de variation & 6,5 & 4,6 \\
\hline \multirow{2}{*}{ Technologies de } & Compost & $75,9^{\mathrm{a}}$ & $6,22^{\mathrm{a}}$ \\
gestion de la & Compost + urée & $64,4^{\mathrm{a}}$ & $5,74^{\mathrm{ab}}$ \\
fertilité du sol & Tiges + urée & $68,8^{\mathrm{a}}$ & $4,92^{\mathrm{bc}}$ \\
& Tiges & $61,6^{\mathrm{a}}$ & $4,39^{\mathrm{c}}$ \\
& Témoin & $70,2^{\mathrm{a}}$ & $4,77^{\mathrm{c}}$ \\
& Probabilité & 0,46 & 0,003 \\
Interaction & Signification & $\mathrm{NS}$ & $\mathrm{HS}$ \\
& Coefficient de variation & 20,4 & 13,9 \\
\hline
\end{tabular}

Dans chaque groupe (macrofaune et technologies de gestion de la fertilité), les moyennes suivies de la même lettre ne diffère pas au seuil de 5\% ; NS : Non Significatif ; HS : Hautement Significatif.

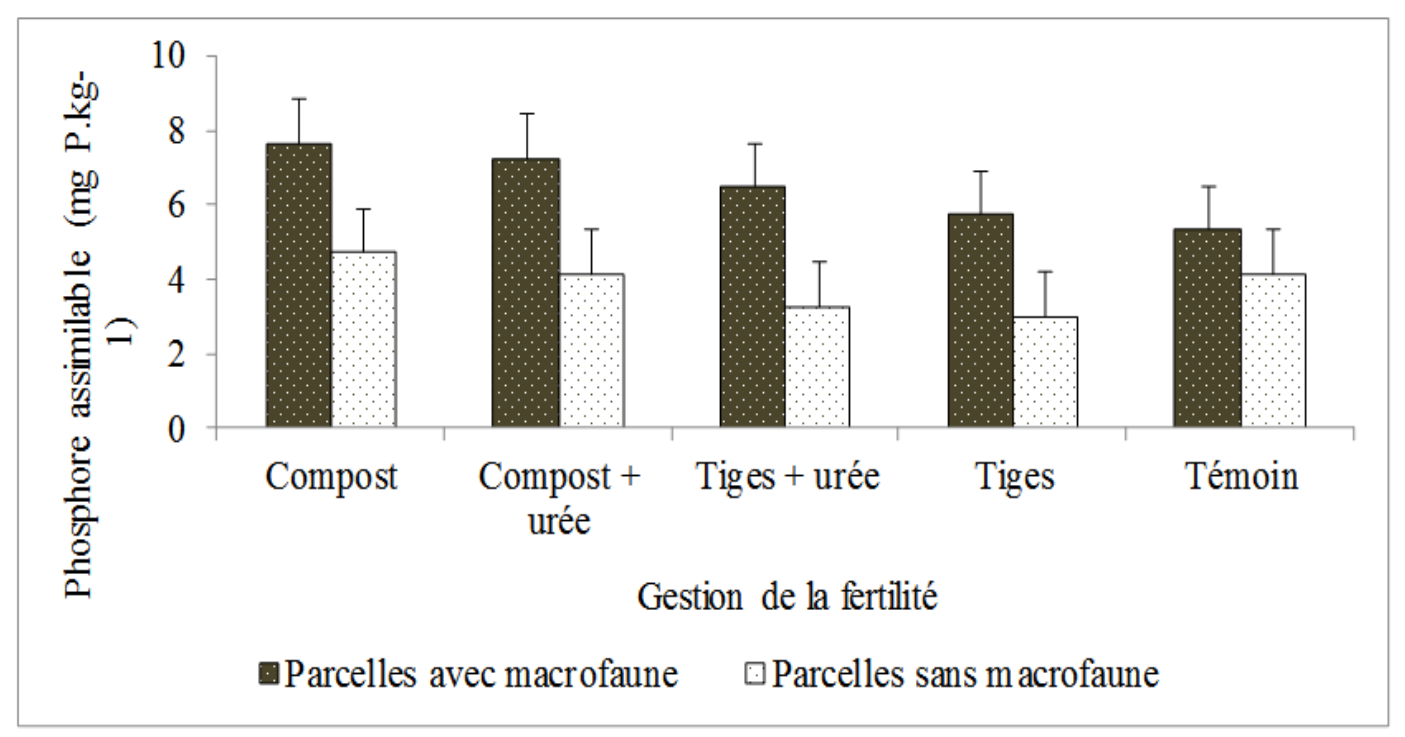

Les barres représentent la plus petite différence significative.

Figure 1 : Teneur en phosphore assimilable du sol des parcelles avec et sans macrofaune du sol. 


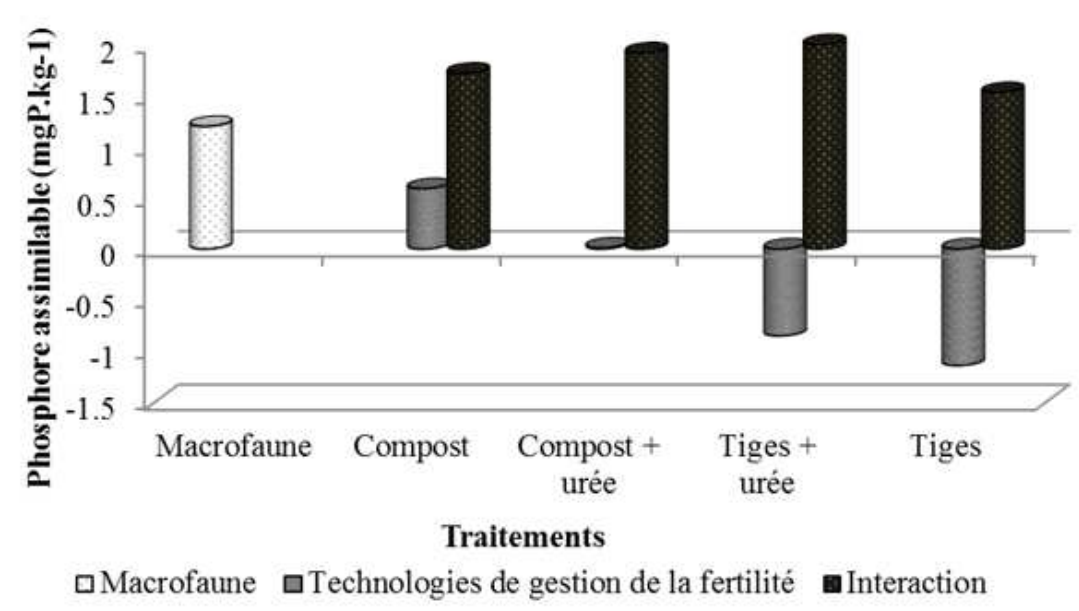

Figure 2 : Effet de la macrofaune, des technologies de gestion de la fertilité et de leur interaction sur la teneur en phosphore assimilable.

\section{DISCUSSION}

Effet de la macrofaune sur le phosphore assimilable du sol

Les résultats ont montré une augmentation significative de la teneur en phosphore assimilable sur les parcelles avec macrofaune comparativement aux parcelles traitées aux pesticides (parcelles sans macrofaune), et ont révélé que la présence de la macrofaune seule améliore la teneur en phosphore assimilable. Ce résultat montre bien que les interactions entre les organismes dans le sol influence la fertilité des sols (Le Bayon et Milleret, 2009). En effet, il a été montré que les turricules de vers ont des teneurs en phosphore plus élevées que le sol environnant (Ouédraogo et al., 2005 ; Kuczak et al., 2006; Chapuis-Lardy et al., 2009 ; Bhadauria et Saxena, 2010). Selon plusieurs auteurs cités par Kuczak et al. (2006), l'augmentation de la teneur en phosphore assimilable dans les turricules s'expliquerait par le $\mathrm{pH}$ élevé dans le tube digestif des vers de terre (Barois et Lavelle, 1986), l'augmentation de l'activité biologique (Lopez-Hernandez et al., 1993), et la sécrétion de mucus riche en composés glucidiques qui contiennent des groupes carboxyle. Ces groupes carboxyle peuvent bloquer les sites de fixation des orthophosphates dans le sol, entraînant de ce fait une augmentation de la teneur en phosphore soluble (LopezHernandez et al., 1993). En outre, la macrofaune du sol régule l'activité, l'abondance des microorganismes, la transformation et le recyclage de la matière organique et des nutriments. Une augmentation de la biomasse des bactéries (Kumari et Sinha, 2012), de la biomasse et du nombre des champignons (Marhan, 2004) suite à l'ingestion d'un sol par les vers de terre ont été observée. Ainsi, les microorganismes interviennent dans la disponibilité du phosphore à travers l'accumulation et le turnover du phosphore de la biomasse microbienne, la minéralisation du phosphore organique et la solubilisation du phosphore inorganique (Richardson et Simpson, 2011). Il est également connu que l'activité des termites entraîne un enrichissement du sol en éléments nutritifs grâce à la remontée d'éléments fins, à la collecte, et à la minéralisation de débris végétaux (LópezHernández et al., 2006). 
Effet des technologies de gestion de la fertilité sur le phosphore assimilable du sol

Les technologies de gestion de la fertilité ont eu un impact hautement significatif sur le phosphore assimilable du sol. Les plus fortes teneurs ont été observées avec le compost seul ou combiné à l'urée. Le compost seul a augmenté le phosphore assimilable de $23 \%$ par rapport au témoin. Ces résultats corroborent ceux de (Soma, 2010) qui ont révélé que, contrairement aux pailles, l'apport de compost augmentait de façon significative la disponibilité du phosphore dans le sol. L'effet du compost sur le phosphore assimilable peut s'expliquer par le fait que le compost contient du phosphore minéral (Traoré et al., 2001) qui augmente la teneur en phosphore assimilable à court terme. A moyen terme, la minéralisation du phosphore organique par les microorganismes induit également une augmentation de la teneur en phosphore assimilable. Par contre, la minéralisation des tiges, beaucoup plus lente à cause de leur rapport $\mathrm{C} / \mathrm{N}$ élevé, n'a pas eu un effet significatif sur le phosphore assimilable du sol. Le calcul de la contribution absolue des technologies de gestion de la fertilité, montre en effet que l'apport de tiges de sorgho a un effet négatif sur la disponibilité de phosphore assimilable. L'apport de matière organique de bonne qualité réduirait la sorption du phosphore, augmentant de ce fait sa disponibilité. L'ajout d'urée aux tiges qui contribue à la minéralisation de la matière a réduit l'effet négatif de l'enfouissement des tiges.

Effet de l'interaction entre les technologies de gestion de la fertilité et la macrofaune sur le phosphore assimilable du sol

Une interaction positive a été révélée entre les technologies de gestion de la fertilité et la macrofaune sur le phosphore assimilable. La présence de la macrofaune, seule ou combinée avec les modes de gestion de la fertilité du sol, se traduit par une augmentation de la teneur en phosphore assimilable. La macrofaune $\mathrm{du}$ sol, en particulier les vers de terre, améliorent donc la disponibilité du phosphore après un apport de composés organiques, confirmant les travaux d'autres auteurs (Ouédraogo et al., 2005 ; Chapuis-Lardy et al., 2009, 2011). Ce résultat montre la synergie entre la macrofaune et les technologies de gestion de la fertilité des sols dans l'amélioration de la biodisponibilité du phosphore dans le sol. La plus forte interaction avec les tiges en présence d'urée révèle l'importance et le rôle de la macrofaune et l'urée dans la décomposition et la minéralisation de substrats organique à rapport $\mathrm{C} / \mathrm{N}$ élevé.

\section{Conclusion}

La macrofaune du sol joue au sein de l'écosystème des rôles essentiels pour le maintien de la qualité des sols. Les résultats montrent que la présence de la macrofaune du sol a augmenté de $68 \%$ la teneur en phosphore assimilable du sol, mais sans augmenter de façon significative, la teneur en phosphore total. Concernant les technologies de gestion de la fertilité des sols, l'apport de compost, seul ou combiné à l'urée, améliore la disponibilité du phosphore. Une interaction positive entre la macrofaune et les technologies de gestion de la fertilité des sols a été mise en évidence. L'interaction a été plus forte avec la technologie combinant les tiges de sorgho et l'urée, révélant ainsi l'importance de la macrofaune dans la minéralisation de la matière organique à rapport $\mathrm{C} / \mathrm{N}$ élevé. La préservation de la diversité de la macrofaune du sol est alors une clé pour une gestion durable des sols dans les systèmes de culture et l'amélioration des rendements. Dans les systèmes de culture à faibles intrants, la stimulation de l'activité de la macrofaune par des apports organiques est nécessaire pour la préservation des potentialités des terres agricoles. Il faut donc repenser les stratégies de protection des cultures, en réduisant et en utilisant de façon judicieuse les pesticides chimiques de synthèse. Les producteurs doivent en effet, adopter les biopesticides et les pesticides 
naturels afin de préserver la diversité biologique des sols agricoles.

\section{REMERCIEMENTS}

Cette étude a été réalisée grâce à l'appui financier de la Fondation Internationale de Science (IFS). Nous remercions également Monsieur OUANDAOGO Noufou, responsable technique du laboratoire Sol-Eau-Plante de Kamboinsé.

\section{REFERENCES}

Bhadauria T, Saxena KG. 2010. Role of earthworms in soil fertility maintenance through the production of biogenic structures. Review Article. Applied and Environmental Soil Science, 2010: 1-7.

Chapuis-Lardy L, Le Bayon R-C, Brossard M, López-Hernández D, Blanchard E. 2011. Role of soil macrofauna in phosphorus cycling. In Soil Biology: Phosphorus in Action: Biological Processes in Soil Phosphorus Cycling, Bünemann EK, Oberson A, Frossard E (eds). Springer Berlin Heidelberg, 199-213.

Chapuis-Lardy L, Ramiandrisoa RS, Randriamanantsoa L, Morel C, Rabeharisoa L, Blanchart E. 2009. Modification of $\mathrm{P}$ availability by endogeic earthworms (Glossoscolecidae) in Ferralsols of the Malagasy Highlands. Biol. Fert. Soils, 45(4): 415-422.

Doré T, Makowski D, Malézieux E, MunierJolaind N, Tchamitchiane M, Tittonell P. 2011. Facing up to the paradigm of ecological intensification in agronomy: Revisiting methods, concepts and knowledge. Review. Eur. J. Agron., 34: 197-210.

Kiba DI, Lompo F, Compaoré E, Randriamanantsoa L, Sedogo PM, Frossard E. 2012. A decade of non-sorted solid urban waste inputs safely increases sorghum yield in the periurban areas of Burkina Faso. Acta Agriculturae Scandinavica Section B-Soil Plant Sci., 62(1): 59-69.
Kuczak CN, Fernandes ECM, Lehmann J, Rondon MA, Luizão FJ. 2006. Inorganic and organic phosphorus pools in earthworm casts (Glossoscolecidae) and a Brazilian rainforest Oxisol. Soil Biol. Biochem., 38: 553-560.

Kumari S, Sinha MP. 2012. Fertility measures of biogenic structures in particular reference to bacterial community. Ann. Biol. Res., 3(9): 4497- 4502.

Lavelle P, Decaëns T, Aubert M, Barot S, Blouin M, Bureau F, Margerie P, Mora P, Rossi JP. 2006. Soil invertebrates and ecosystem services. Eur. J. Soil Biol., 42: S3-S15.

Le Bayon R-C, Milleret R. 2009. Effects of earthworms on phosphorus dynamic - a review. Dynamic Soil, Dynamic Plant, 3(2): 21-27.

Li X, Ji R, Schäffer A, Brune A. 2006. Mobilization of soil phosphorus during passage through the gut of larvae of Pachnoda ephippiata (Coleoptera: Scarabaeidae). Plant Soil, 288: 263-270.

Lompo F, Segda Z, Gnankambary Z, Ouandaogo N. 2009. Influence des phosphates naturels sur la qualité et la bio-dégradation d'un compost de pailles de maïs. Tropicultura, 27(2): 105-109.

Lompo F. 2009. Effets induits des modes de gestion de la fertilité sur les états du phosphore et la solubilisation des phosphates naturels dans deux sols acides du Burkina Faso. Thèse doctorat d'Etat, Université de Cocody, Côte d'Ivoire, p. 254.

López-Hernández, Brossard M, Fardeau JC, Lepage M. 2006. Effect of different termite feeding groups on $\mathrm{P}$ sorption and $P$ availability in African and South American savannas. Biol. Fert. Soils, 42: 207-214.

Marhan S. 2004. Effects of earthworms on stabilisation and mobilisation of soil organic matter. Dissertation, Fachbereich Biologie der Technischen Universität Darmstadt, p. 160. 
Nonguierma GB. 2006. Contribution à l'évaluation des effets de l'utilisation des pesticides en production maraîchère dans la plaine périurbaine de BoulmiougouOuagadougou. Mémoire de Licence Professionnelle en Génie de l'Environnement. Université de Ouagadougou, Unité de Formation et de Recherche en Sciences Exactes et Appliquées. Institut de Génie de l'Environnement et du Développement durable, Burkina Faso, p. 45.

Ouédraogo E, Brussaard L, Mando A, Stroosnijder L. 2005. Organic resources and earthworms affect phosphorus availability to sorghum after phosphate rock addition in semi-arid West Africa. Biol. Fert. Soils, 41: 458-465.

Ouédraogo E, Brussaard L, Stroosnijder L. 2007. Soil macrofauna and organic amendment interactions affects soil carbon and crop performance in semi-arid West Africa. Biol. Fert. Soils, 44(2): 343351 .

Petit M. 2011. Pour Une Agriculture Productive et Durable. Editions Quae, Versailles: France.

Richardson AE, Simpson RJ. 2011. Soil organisms mediating phosphorus availability. Plant Physiol., 156: 989-996.

Soma DM. 2010. Effet des apports répétés de diverses sources d'amendements organiques dans un sol ferrugineux tropical lessivé (Saria, Burkina Faso) sur la biodisponibilité du phosphore et la production du sorgho. Mémoire de DEA, Université polytechnique de BoboDioulasso, Burkina Faso, p. 47.

Traoré M. 2012. Impact des pratiques agricoles (rotation, fertilisation et labour) sur la dynamique de la microfaune et la macrofaune du sol sous culture de sorgho et de niébé au Centre Ouest du Burkina Faso. Thèse de doctorat unique en science du sol, Université Polytechnique de Bobo Dioulasso, Burkina Faso, p. 147.

Traoré O, Sinaj S, Frossard E. 2001. Disponibilité du phosphore de composts issus de déchets organiques pour le trèfle blanc (Trifolium repens). Cah. Agric., 10(6): 389-396.

Vall E, Koutou M, Blanchard M, Koulibaly K, Diallo AM, Andrieu N. 2012. Intégration agriculture-élevage et intensification écologique dans les systèmes agrosylvopastoraux de l'Ouest du Burkina Faso, province du Tuy. Actes du séminaire, novembre 2011, BoboDioulasso, Burkina Faso, p. 13.

WRB (World Reference Base for Soil Resources). 2006. A framework for International Classification, Correlation and Communication. FAO, Rome, Italie.

Zida Z, Ouédraogo E, Mando A, Stroosnijder L. 2011. Termite and earthworm abundance and taxonomic richness under long-term conservation soil management in Saria, Burkina Faso, West Africa. Appl. Soil Ecol., 51: 122-129. 